ISSN 0103-9954

\title{
INFLUÊNCIA DA TEMPERATURA NA RESISTÊNCIA E NO MÓDULO DE ELASTICIDADE EM MADEIRA DE HÍBRIDOS DE EUCALIPTOS
}

\author{
INFLUENCE OF TEMPERATURE IN THE STRENGTH AND MODULUS OF ELASTICITY IN \\ WOOD FROM HYBRIDS OF EUCALYPTUS
}

\author{
Edgar Vladimiro Mantilla Carrasco ${ }^{1}$ Ana Lúcia Crespo Oliveira ${ }^{2}$ Judy Norka Rodo Mantilla ${ }^{3}$
}

\begin{abstract}
RESUMO
$\mathrm{O}$ trabalho trata da influência da temperatura, em um intervalo de $20^{\circ} \mathrm{C}$ a $24^{\circ} \mathrm{C}$, nas propriedades físicas da madeira: umidade e massa específica e mecânicas: resistência e módulo de elasticidade à compressão paralela às fibras. O programa experimental foi realizado com 78 corpos de prova, de híbridos de Eucalyptus grandis com Eucalyptus urophylla, com massa específica aparente média de $671 \mathrm{~kg} / \mathrm{m}^{3}$ e teor de umidade de $11,14 \%$. Foram seguidos os procedimentos dos ensaios prescritos pela NBR 7190. Os resultados mostram uma diminuição linear do teor de umidade com o aumento da temperatura até $160^{\circ} \mathrm{C}$. Destacamse duas retas, a primeira de $20^{\circ} \mathrm{C}$ até $65^{\circ} \mathrm{C}$ com pequeno decréscimo do teor de umidade, chegando a $4 \%$ e a segunda de $65^{\circ} \mathrm{C}$ até $160^{\circ} \mathrm{C}$ com perda mais acentuada chegando a $0 \%$ de teor de umidade. Acima de $160^{\circ} \mathrm{C}$, não apresentando mais umidade, a madeira perde apenas material orgânico, chegando a $20 \%$ de perda de massa quando atinge uma temperatura de $240^{\circ} \mathrm{C}$. A massa específica praticamente permanece constante até a temperatura de $100^{\circ} \mathrm{C}$. A partir da temperatura de $100^{\circ} \mathrm{C}$ até $240^{\circ} \mathrm{C}$ a massa específica decresce linearmente chegando a $20 \%$. A resistência à compressão paralela às fibras aumenta linearmente com a temperatura, chegando a $19 \%$ na temperatura de $100^{\circ} \mathrm{C}$ e, após esta temperatura, o comportamento se inverte com decréscimo linear até a temperatura de $180^{\circ} \mathrm{C}$. A partir de $180^{\circ} \mathrm{C}$, a queda de resistência é acentuada, chegando, na temperatura de $240^{\circ} \mathrm{C}$, a $32 \%$ menor do que a resistência na temperatura de $20^{\circ} \mathrm{C}$. $\mathrm{O}$ módulo de elasticidade diminui, apenas aumenta a temperatura. Até $120^{\circ} \mathrm{C}$ existe uma diminuição linear não muito acentuada, em torno de $8 \%$. Já de $120^{\circ} \mathrm{C}$ até $200^{\circ} \mathrm{C}$ a diminuição também é linear, porém, bastante acentuada chegando a $38 \%$.
\end{abstract}

Palavras-chave: altas temperaturas; degradação térmica da madeira; resistência da madeira.

\begin{abstract}
This paper deals with the influence of temperature in a range from $20^{\circ} \mathrm{C}$ to $240{ }^{\circ} \mathrm{C}$, in the physical properties of wood: humidity and specific and mechanical masses: strength and modulus of elasticity for the parallel compression to the fibers. The experimental program was performed with 78 specimens of hybrids of Eucalyptus grandis x Eucalyptus urophylla with mean apparent specific gravity of $671 \mathrm{~kg} / \mathrm{m}^{3}$ and moisture content of $11.14 \%$. The test procedures prescribed by NBR 7190 were followed. The results show a linear decrease in moisture content with increasing temperature up to $160^{\circ} \mathrm{C}$. Two straight lines are noteworthy, the first one from $20^{\circ} \mathrm{C}$ to $65^{\circ} \mathrm{C}$ with slight decrease in moisture content, reaching $4 \%$ and the second one from $65^{\circ} \mathrm{C}$ to $160^{\circ} \mathrm{C}$ with the sharpest loss reaching $0 \%$ content of moisture. Above $160^{\circ} \mathrm{C}$, the moisture content is zero, so that the wood loses only organic material, coming to lose $20 \%$ by weight when it reaches

1 Engenheiro Civil, Dr., Professor Titular do Departamento de Engenharia de Estruturas, Escola de Engenharia, Universidade Federal de Minas Gerais, Av. Antonio Carlos, 6627, Campus Pampulha, Bloco 1, $4^{\circ}$ Andar, Sala 4123, CEP 31270-901, Belo Horizonte (MG), Brasil. mantilla@dees.ufmg.br

2 Engenheira Civil, Msc., Doutoranda no Departamento de Engenharia de Estruturas, Escola de Engenharia, Universidade Federal de Minas Gerais, Av. Antonio Carlos, 6627, Campus Pampulha, Bloco 1, $4^{\circ}$ Andar, Sala 4123, CEP 31270-901, Belo Horizonte (MG), Brasil. lucia@dees.ufmg.br

3 Engenheira Civil, Dra ${ }^{\mathrm{a}}$, Professora Assistente da Faculdade de Engenharia e Arquitetura, Universidade FUMEC, Rua Dom Modesto Augusto, 102, Apto. 200, Coração Eucarístico, CEP 30535-430, Belo Horizonte (MG), Brasil. judy.mantilla@fumec.br
\end{abstract}

Recebido para publicação em 30/10/2012 e aceito em 24/09/2014

Ci. Fl., v. 26, n. 2, abr.-jun., 2016 
a temperature of $240^{\circ} \mathrm{C}$. The specific mass remains practically constant until the temperature of $100^{\circ} \mathrm{C}$. Temperature between $100^{\circ} \mathrm{C}$ to $240^{\circ} \mathrm{C}$, the specific mass decreases linearly reaching 20 per cent. The strength to compression parallel to the fibers increases linearly with the temperature, reaching $19 \%$ at $100^{\circ} \mathrm{C}$. Above this temperature, the behavior is inverse, with linear decrease until the temperature of $180^{\circ} \mathrm{C}$. From $180^{\circ} \mathrm{C}$ the compression strength decreases reaching the temperature of $240^{\circ} \mathrm{C}$ at $32 \%$ lower than the resistance at the temperature of $20^{\circ} \mathrm{C}$. As the modulus of elasticity decreases, it only increases the temperature. Up to the temperature of $120^{\circ} \mathrm{C}$, it has a very slight linear decrease of around 8 per cent. However, between $120^{\circ} \mathrm{C}$ and $200^{\circ} \mathrm{C}$ the decrease is also linear, but more accentuated, reaching 38 per cent.

Keywords: high temperatures; thermal degradation of wood; strength of wood.

\section{INTRODUÇÃO}

As mudanças no comportamento dos elementos estruturais quando submetidos a altas temperaturas, principalmente no dimensionamento em situação de incêndio, são devido às mudanças das características mecânicas em função da temperatura, ABNT NBR 14432 (2001). O aumento da temperatura do elemento estrutural provoca a redução das características mecânicas do material, assim como o desenvolvimento de tensões que sobrecarregam a estrutura (COSTA; SILVA, 2006). Manríquez e Moraes (2009), Buchanan (2004), Pinto (2005) e Schaffer (1967) compartilham essas evidências.

Segundo Purkiss (2007), na madeira estrutural sujeita ao fogo, a camada externa carbonizada perde a resistência, mas resguarda a parte interna não carbonizada do excesso de temperatura garantindo assim uma menor perda de resistência do material remanescente. E acrescenta que três propriedades são necessárias para se determinar o comportamento da madeira estrutural ao fogo: a taxa de carbonização, a resistência e a elasticidade da madeira.

A madeira é definida quimicamente como um biopolímero tridimensional composto por uma rede interligada de celulose, hemicelulose, lignina e pequenas quantidades de compostos extraíveis e inorgânicos. As temperaturas elevadas provocam a degradação térmica dos componentes químicos presentes em sua estrutura molecular. Pinheiro e Sèye (1998) e Raad, Pinheiro e Yoshida (2006) verificaram experimentalmente que, dos três principais componentes da madeira, a hemicelulose e a celulose, cerca de $70 \%$ da composição da madeira, são instáveis e se despolimerizam com temperaturas entre $225^{\circ} \mathrm{C}$ e $375^{\circ} \mathrm{C}$. Os autores afirmam que a perda acentuada de massa é devido à degradação desses dois componentes. A lignina, outro elemento constituinte, por ser estável, se degrada de modo mais lento sendo, no entanto, o principal responsável pela carbonização da madeira. Outra abordagem é apresentada por White e Dietenberger (2001) que indicam que o grau das alterações depende dos seguintes fatores: gradiente de temperatura ao qual a madeira é exposta, condições e período de exposição, fonte de aquecimento, teor de umidade e espécie da madeira.

Segundo Siau (1984), por causa de estrutura porosa da madeira, a condutividade térmica é baixa $(\lambda=0,12 \mathrm{~W} / \mathrm{m} . \mathrm{K})$. Esse fato é devido à quantidade de ar presente em seu interior, já que a condutividade térmica do ar é de $\lambda=0,0216 \mathrm{~W} / \mathrm{m} \mathrm{K}$. Diferentemente de materiais homogêneos, o fluxo de calor que ocorre na madeira varia em cada plano anatômico e em função de irregularidades existentes como fendas, nós, etc. No sentido das fibras é 2,25 a 2,75 vezes maior do que nos outros sentidos (radial e tangencial). A constituição física do material lenhoso influencia também na condutividade do calor, quanto maior for a massa específica (menor quantidade de ar por unidade de volume) maior será sua condutividade térmica e quanto maior for o teor de umidade, maior será também a condutividade térmica, visto que a condutividade térmica da água é $\lambda=0,5 \mathrm{~W} / \mathrm{m} \mathrm{K}$.

Skaar (1988) afirma que a água no lenho da madeira, quando a umidade está acima de $28 \%$, encontra-se livree percola pelos vazios doselementos anatômicos da madeira. Já entre $6 \%$ e $28 \%$ de umidade a água ocupa os espaços submicroscópicos da parede celular, em camadas polimoleculares ligadas por forças elétricas polares e entre $0 \%$ e $6 \%$ de umidade, a água é chamada de água de adsorção química e está aderida às interfaces existentes entre moléculas de celulose e de hemicelulose. $\mathrm{O}$ autor comenta, além disso, que a madeira com umidade apresenta comportamento diferenciado quando sujeita à variação da temperatura. Se for aquecida, 
ela tende a se expandir por efeito térmico e a se contrair, devido à perda de umidade. A menos que a madeira esteja com um teor de umidade inicial muito baixo (menor que $3 \%$ ou $4 \%$ de umidade), a contração, em decorrência da perda de umidade, será maior que a expansão térmica, de forma que a mudança dimensional resultante será negativa. No entanto, quando a madeira está com teor de umidade intermediário, entre $8 \%$ e $20 \%$, ao ser aquecida, inicialmente, ela irá se expandir para logo se contrair gradualmente, até um volume menor que o inicial à medida que ocorre a perda de umidade por efeito do aquecimento.

As propriedades de resistência mecânica da madeira variam linearmente com a temperatura, quando submetida a temperaturas abaixo de $150^{\circ} \mathrm{C}$, para um teor de umidade constante (KRETSCHMANN, 2010). A mudança que ocorre nas propriedades quando a madeira é rapidamente aquecida ou esfriada é denominada de "efeito imediato". Em temperaturas abaixo de $100^{\circ} \mathrm{C}$, o "efeito imediato" é reversível, isto é, se a mudança de temperatura for rápida, a propriedade irá retornar para o valor correspondente à temperatura original. Acima dos $100^{\circ} \mathrm{C}$ ocorre o "efeito irreversível" devido à degradação de substâncias componentes da madeira, o que resulta em perda de peso e resistência. Esta perda depende de fatores que incluem teor de umidade, meio de aquecimento, gradiente de temperatura, período de exposição, espécie e tamanho da peça analisada.

Schaffer (1967) comenta que, acima de $100^{\circ} \mathrm{C}$, a água da madeira exposta ao calor evapora, no entanto, uma parte migra para o interior e que resulta em um aumento da umidade na parte interna da madeira.

Durante um incêndio, a camada aquecida de madeira por baixo do carvão que se forma, tem suas propriedades mecânicas reduzidas devido ao aumento de temperatura e à mudança na umidade. No entanto, segundo Piazza et al. (2005), acima de $65^{\circ} \mathrm{C}$ a madeira apresenta uma melhoria das características mecânicas, devido ao fato da umidade diminuir, dessa forma pode ser mascarado o efeito negativo da degradação térmica.

Manriquez (2008, 2012) analisou resultados de diferentes estudos (SCHAFFER 1967, YOUNG; CLANCY, 2001, KNUDSON; SCHNIEWIND， 1975, GERHARDS， 1982, THOMAS, 1996, BAKAR; HIZIROGLU; TAHIR, 2012) com a finalidade de avaliar a influência do aumento de temperatura na perda de resistência à compressão da madeira. Concluiu que a resistência à compressão paralela às fibras dos corpos de prova de madeira seca é maior que a dos corpos de prova de madeira úmida. Da mesma forma, Manríquez e Moraes (2009) analisaram resultados de resistência à compressão paralela às fibras, obtidos por diferentes pesquisadores, em corpos de prova de diferentes espécies submetidos à variação de temperatura. Concluem que a diferença está associada ao teor de umidade das amostras, de forma que menores teores de umidade permitem uma degradação mais rápida dos polímeros da madeira, refletindo na perda de resistência. Também concluíram que sob o efeito da temperatura, de $20^{\circ} \mathrm{C}$ até $210^{\circ} \mathrm{C}$, o módulo de elasticidade diminui cerca de $40 \%$, podendo chegar a uma redução de $90 \%$ para $250^{\circ} \mathrm{C}$.

Manríquez (2008) analisou amostras da espécie paricá (Schizolobium amazonicum) com teor de umidade médio de $11,74 \%$ e massas específicas aparentes entre $282,94 \mathrm{~kg} / \mathrm{m}^{3}$ e $464,53 \mathrm{~kg} / \mathrm{m}^{3}$, submetidas a temperaturas entre $20^{\circ} \mathrm{C}$ e $230^{\circ} \mathrm{C}$ e verificou que a resistência mecânica diminui em função do aumento da temperatura sendo que a $230^{\circ} \mathrm{C}$ essa redução chega a $35,07 \%$ para a resistência à compressão. Também Manríquez e Moraes (2009) analisaram resultados experimentais de diversos pesquisadores e concluíram que ocorreram reduções na resistência à compressão paralela às fibras de até $75 \%$, para diversas espécies de madeira, em gradientes até $240^{\circ} \mathrm{C}$.

Nesse sentido, o objetivo do trabalho é avaliar a influência da variação de temperatura, em um intervalo de $20^{\circ} \mathrm{C}$ a $240^{\circ} \mathrm{C}$, na umidade, massa específica, resistência e módulo de elasticidade à compressão paralela às fibras da madeira.

\section{MATERIAIS E MÉTODOS}

\section{Material}

A madeira utilizada no programa experimental é de híbridos de Eucalyptus grandis com Eucalyptus urophylla oriunda de florestas plantadas no sul da Bahia, Brasil. Foram utilizadas seis vigas de madeira serradas, secas em estufa, com seções 50 x $180 \mathrm{~mm}$ e comprimentos de $3690 \mathrm{~mm}$ com teor de umidade de $11,14 \%$ e massa específica aparente média de $671 \mathrm{~kg} / \mathrm{m}^{3}$. As vigas foram compradas em uma serraria de Belo Horizonte.

De cada uma das 6 vigas foram retirados 14 corpos de prova (CPs) de 50 × 50 × $150 \mathrm{~mm}$, sendo a dimensão de $150 \mathrm{~mm}$ paralela às fibras conforme 
a NBR 7190 (1997), o que resultou um total de 84 CPs. Foram formados 12 conjuntos de $7 \mathrm{CPs}$, sendo 6 de cada viga e $1 \mathrm{CP}$ para acompanhamento de cada temperatura, retirado aleatoriamente de uma das vigas. Os conjuntos de CPs foram submetidos à temperatura ambiente em uma câmara climatizada a $20^{\circ} \mathrm{C}$ e umidade relativa $68 \%$, pelo período de 3 messes. Nove destes conjuntos foram acondicionados em estufas com circulação de ar, nas temperaturas $40^{\circ} \mathrm{C}, 60^{\circ} \mathrm{C}, 80^{\circ} \mathrm{C}, 100^{\circ} \mathrm{C}, 120^{\circ} \mathrm{C}$, $140^{\circ} \mathrm{C}, 160^{\circ} \mathrm{C}, 180^{\circ} \mathrm{C}$ e $200^{\circ} \mathrm{C}$. Dois conjuntos foram acondicionados em forno mufla nas temperaturas de $220^{\circ} \mathrm{C}$ e $24^{\circ} \mathrm{C}$.

\section{Metodologia de ensaio}

De cada conjunto de sete CPs, o CP para acompanhamento do gradiente de temperatura (testemunha) foi previamente perfurado na parte superior para a instalação de um sensor de temperatura (termopar tipo K). Uma vez inserido no furo, o sensor foi protegido por um tubo de cerâmica refratária. A variação de temperatura no interior do corpo de prova (CP) foi acompanhada por um sistema de aquisição de dados (Figura 1). Quando a câmara quente (estufa ou forno mufla) atingiu a temperatura definida para cada conjunto, o tempo de permanência dos CPs, nessa temperatura, foi definido pelo sensor de temperatura embutido no $\mathrm{CP}$ para acompanhamento do gradiente de temperatura. Quando este sensor atingia a temperatura da câmara quente, indicava que toda a massa dos CPs também atingiram esta temperatura. Em seguida

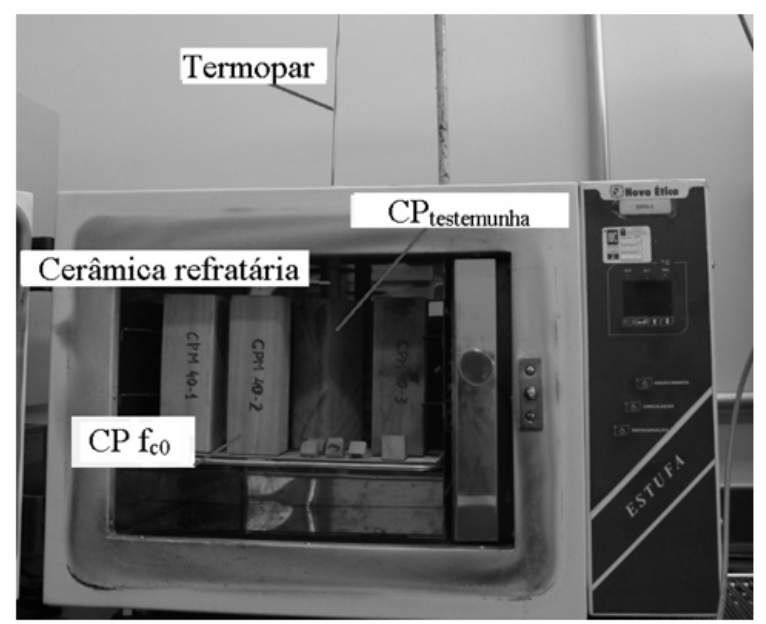

FIGURA 1: Condicionamento do CP na câmara de temperatura.

FIGURE 1: Conditioning of specimens in chamber temperature. foram retirados os CPs da câmara quente. A taxa de aquecimento foi de $5^{\circ} \mathrm{C}$ por minuto.

Antes de serem colocados os CPs na câmara quente, determinavam-se peso e dimensões de cada $\mathrm{CP}$, para a obtenção da umidade e massa específica aparente, segundo os procedimentos da ABNT NBR 7190 (1997), anexo B. Essa mesma norma foi adotada para a determinação da resistência à compressão e do módulo de elasticidade paralelo às fibras. Devido às dimensões dos CPs serem bem maiores que os indicados pela norma e para determinação do volume foram feitas 5 medidas de cada aresta, a utilização do método estereométrico foi considerado suficientemente válido.

Os ensaios foram realizados em uma Máquina Universal com capacidade de $30 \mathrm{kN}$. Para a medida de deformação foi utilizado um clipgage com comprimento base de $50 \mathrm{~mm}$ (Figura 2). O carregamento aplicado foi de acordo com o diagrama de carga (cíclico) adotado pela ABNT NBR 7190 (1979).

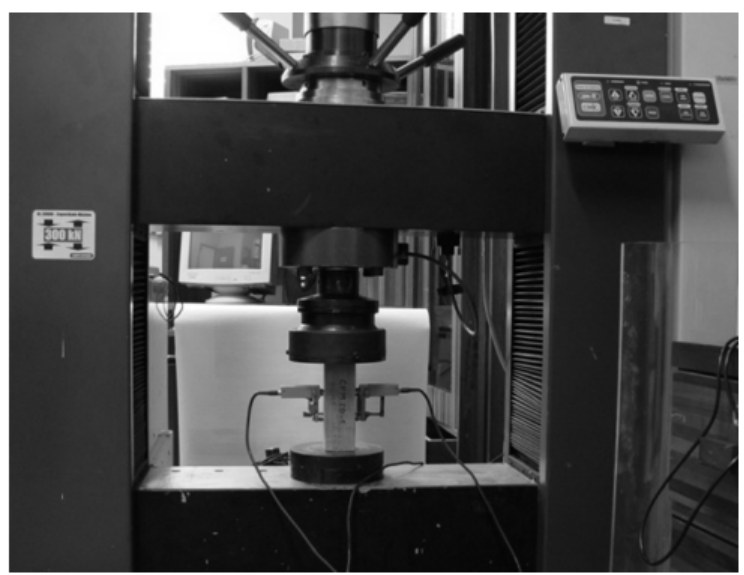

FIGURA 2: Ensaio à compressão.

FIGURE 2: Test compression.

\section{RESULTADOS E DISCUSSÃO}

\section{Definições}

Foi definida a resistência relativa à compressão paralela às fibras, como a razão entre a resistência à compressão paralela as fibras, em uma determinada temperatura $(\mathrm{T})$ e a resistência à compressão paralela às fibras a $20^{\circ} \mathrm{C}$, representada por $\mathrm{f}_{\mathrm{c} 0}{ }^{\circ} \mathrm{T} / \mathrm{f}_{\mathrm{co}^{\circ} 20^{\circ} \mathrm{C}}$. E o módulo de elasticidade relativo paralelo às fibras como a razão entre o módulo de elasticidade paralelo às fibras, em uma dada temperatura (T) e o módulo de elasticidade paralelo às fibras a $20^{\circ} \mathrm{C}$, representada por $\mathrm{E}_{\mathrm{c} 0{ }^{\mathrm{T}}} / \mathrm{E}_{\mathrm{c} 0{ }^{\prime} 0^{\circ} \mathrm{C}}$. $\mathrm{A}$ 
massa específica relativa como a razão entre a massa específica aparente em uma dada temperatura (T) e a massa específica aparente a $20^{\circ} \mathrm{C}$, representada por $\mathrm{D}_{\mathrm{ap}, \mathrm{T}} / \mathrm{D}_{\mathrm{ap}, 20^{\circ} \mathrm{C}}$.

\section{Teor de umidade e massa específica aparente}

O teor de umidade médio das 6 vigas selecionadas à temperatura de $20^{\circ} \mathrm{C}$, foi de $11,18 \%$. Após a retirada dos CPs da câmara quente e a realização dos ensaios mecânicos, foi determinado seu teor de umidade, que está apresentado na Tabela 1 para todas as temperaturas de ensaio. Notar que os valores "negativos de umidade" (valores com * na Tabela 1) são referentes à perda de massa de outros componentes da madeira e não mais de água.

Na Figura 3 é mostrada a variação do teor de umidade em função do aumento da temperatura.

É importante ressaltar que o tempo de permanência, na temperatura, foi o necessário para que toda a massa do CP ficasse com a mesma temperatura. Na Figura 3 destacam-se duas retas, a primeira de $20^{\circ} \mathrm{C}$ até $65^{\circ} \mathrm{C}$ e a segunda de $65^{\circ} \mathrm{C}$ até $160^{\circ} \mathrm{C}$, com inclinações diferentes e $\mathrm{R}^{2}$ de 0,85 e 0,95 , respectivamente. Com relação à primeira reta, a variação da umidade em função da temperatura, até aproximadamente $65^{\circ} \mathrm{C}$, é de $4 \%$. Kretschmann (2010) indica que, até essa temperatura, as mudanças que ocorrem na madeira, sob a influência da temperatura, são reversíveis ("efeito imediato") e para temperaturas maiores que $65^{\circ} \mathrm{C}$ as mudanças são devidas a processos químicos, assim a desidratação da madeira nesta etapa ocorre por perda de água adsorvida.

Observa-se na Tabela 1, que, quando o CP está numa temperatura entre $140^{\circ} \mathrm{C}$ e $180^{\circ} \mathrm{C}$, o teor de umidade poderá ser zero. Já na Figura 3, podese constatar que, quando o $\mathrm{CP}$ atinge a temperatura de $160^{\circ} \mathrm{C}$, o teor de umidade é zero. Acima dessa temperatura o $\mathrm{CP}$ começa a perder os componentes orgânicos da madeira, isto explica o valor "negativo da umidade". Esse fato também foi observado por Pinheiro e Sèye (1998), porém, com temperaturas acima de $200^{\circ} \mathrm{C}$, o que confirma a degradação dos componentes mais instáveis da madeira: a hemicelulose e a celulose.

A massa específica aparente de cada um dos CPs é apresentada na Tabela 2 em função da temperatura, e na Figura 4 a massa específica relativa em função da temperatura.

A massa específica relativa é próxima de um até a temperatura de $100^{\circ} \mathrm{C}$ (Figura 4), o que significa que a massa específica da madeira submetida até essa temperatura apresenta um valor igual ao da massa específica na temperatura ambiente de $20^{\circ} \mathrm{C}$, indicando que a perda de volume devido à

TABELA 1: Teor de umidade dos corpos de prova em função da temperatura.

TABLE 1: Moisture content of specimens because of the temperature.

\begin{tabular}{ccccccccc}
\hline \multirow{2}{*}{ Temp. $\left({ }^{\circ} \mathrm{C}\right)$} & $\mathrm{U}_{\mathrm{f}}(\%)$ & $\mathrm{U}_{\mathrm{f}}(\%)$ & $\mathrm{U}_{\mathrm{f}}(\%)$ & $\mathrm{U}_{\mathrm{f}}(\%)$ & $\mathrm{U}_{\mathrm{f}}(\%)$ & $\mathrm{U}_{\mathrm{f}}(\%)$ & $\mathrm{U}_{\mathrm{f}}(\%)$ & $\mathrm{U}_{\mathrm{f}}(\%)$ \\
\cline { 2 - 8 } & Viga 1 & Viga 2 & Viga 3 & Viga 4 & Viga 5 & Viga 6 & Média & Desv.Pad. \\
\hline 20 & 11,14 & 11,20 & 11,17 & 11,14 & 11,24 & 11,19 & 11,18 & 0,04 \\
40 & 10,81 & 10,60 & 10,75 & 10,74 & 11,01 & 9,67 & 10,60 & 0,47 \\
60 & 9,90 & 9,50 & 9,81 & 9,61 & 10,14 & 8,81 & 9,63 & 0,46 \\
80 & 7,01 & 7,25 & 7,22 & 6,31 & 7,23 & 6,66 & 6,95 & 0,38 \\
100 & 6,00 & 5,97 & 5,99 & 5,47 & 5,73 & 5,03 & 5,70 & 0,39 \\
120 & 3,04 & 3,31 & 3,85 & 2,26 & 4,10 & 3,70 & 3,38 & 0,67 \\
140 & 1,88 & 1,63 & 2,12 & 1,09 & 2,10 & 1,03 & 1,64 & 0,48 \\
160 & 0,71 & $-0,04^{1}$ & 0,38 & $-0,07^{1}$ & 0,09 & $-1,63^{1}$ & $-0,09^{1}$ & 0,81 \\
180 & $-0,45^{1}$ & $-1,72^{1}$ & $-1,35^{1}$ & $-1,24^{1}$ & $-1,91^{1}$ & $-4,30^{1}$ & $-1,83^{1}$ & 1,31 \\
200 & $-8,94^{1}$ & $-6,41^{1}$ & $-7,89^{1}$ & $-7,94^{1}$ & $-3,41^{1}$ & $-8,71^{1}$ & $-7,22^{1}$ & 2,06 \\
220 & $-10,32^{1}$ & $-11,30^{1}$ & $-11,72^{1}$ & $-13,16^{1}$ & $-11,79^{1}$ & $-13,61^{1}$ & $-11,98^{1}$ & 1,21 \\
240 & $-15,98^{1}$ & $-13,81^{1}$ & $-14,69^{1}$ & $-14,33^{1}$ & $-11,25^{1}$ & $-15,00^{1}$ & $-14,18^{1}$ & 1,61 \\
\hline
\end{tabular}

Em que: 'significa que o valor não é mais referente à umidade e sim à perda de componentes da madeira, $\mathrm{U}_{\mathrm{f}}=$ umidade final. 


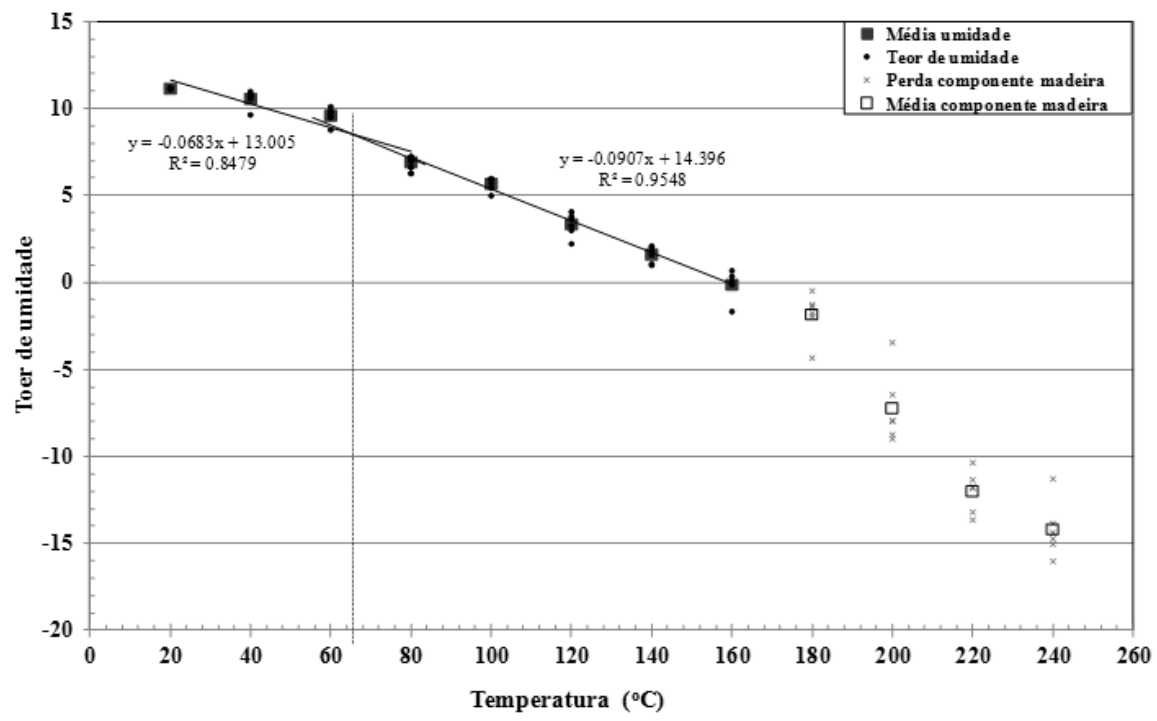

FIGURA 3: Teor de umidade em função do aumento da temperatura.

FIGURE 3: Moisture content versus increasing temperature.

TABELA 2: Massa específica aparente dos corpos de prova em função da temperatura.

TABLE 2: Specific Gravity as a function of temperature in the specimens.

\begin{tabular}{ccccccccc}
\hline \multirow{2}{*}{ Temperatura $\left({ }^{\circ} \mathrm{C}\right)$} & $\begin{array}{c}\mathrm{D}_{\text {ap }} \\
\text { Viga 1 }\end{array}$ & $\begin{array}{c}\mathrm{D}_{\text {ap }} \\
\text { Viga 2 }\end{array}$ & $\begin{array}{c}\mathrm{D}_{\text {ap }} \\
\text { Viga 3 }\end{array}$ & $\begin{array}{c}\mathrm{D}_{\text {ap }} \\
\text { Viga 4 }\end{array}$ & $\begin{array}{c}\mathrm{D}_{\text {ap }} \\
\text { Viga 5 }\end{array}$ & $\begin{array}{c}\mathrm{D}_{\text {ap }} \\
\text { Viga 6 }\end{array}$ & $\begin{array}{c}\mathrm{D}_{\text {ap }} \\
\text { Média }\end{array}$ & $\begin{array}{c}\mathrm{D}_{\text {ap }} \\
\text { Desv.Pad. }\end{array}$ \\
\hline 20 & 678 & 750 & 710 & 583 & 714 & 740 & 696 & 61 \\
40 & 675 & 748 & 709 & 579 & 705 & 745 & 694 & 62 \\
60 & 674 & 719 & 708 & 591 & 710 & 769 & 695 & 60 \\
80 & 674 & 718 & 733 & 577 & 711 & 765 & 696 & 66 \\
100 & 672 & 731 & 757 & 588 & 700 & 743 & 699 & 62 \\
120 & 590 & 702 & 730 & 576 & 678 & 738 & 669 & 70 \\
140 & 563 & 688 & 718 & 570 & 668 & 737 & 657 & 74 \\
160 & 537 & 674 & 706 & 563 & 657 & 736 & 646 & 79 \\
180 & 510 & 660 & 694 & 557 & 647 & 735 & 634 & 85 \\
200 & 529 & 628 & 651 & 537 & 623 & 675 & 607 & 60 \\
220 & 528 & 609 & 626 & 497 & 615 & 633 & 585 & 57 \\
240 & 491 & 590 & 605 & 494 & 580 & 640 & 567 & 61 \\
\hline
\end{tabular}

Em que: $\mathrm{D}_{\mathrm{ap}}=$ Massa específica aparente.

diminuição da umidade é compensada pelo aumento de volume devido à dilatação térmica. Na Figura 4 e na Tabela 2, observa-se uma redução crescente da densidade de $5 \%$ a $20 \%$, respectivamente para uma temperatura de $120^{\circ} \mathrm{C}$ a $240^{\circ} \mathrm{C}$. Esta redução ocorreu por perda de água, por expansão térmica e por perda de material orgânico, no presente trabalho não foi possível definir especificamente as proporções de cada fenômeno. Segundo Skaar (1988), o aumento da temperatura provoca uma diminuição da densidade da madeira, principalmente, devido à perda de água presente nas interfaces entre moléculas de celulose e hemicelulose, rompendose as forças intermoleculares de Van der Waals e as Ligações de Hidrogênio.

$\mathrm{Na}$ Figura 5 é apresentado um gráfico da 


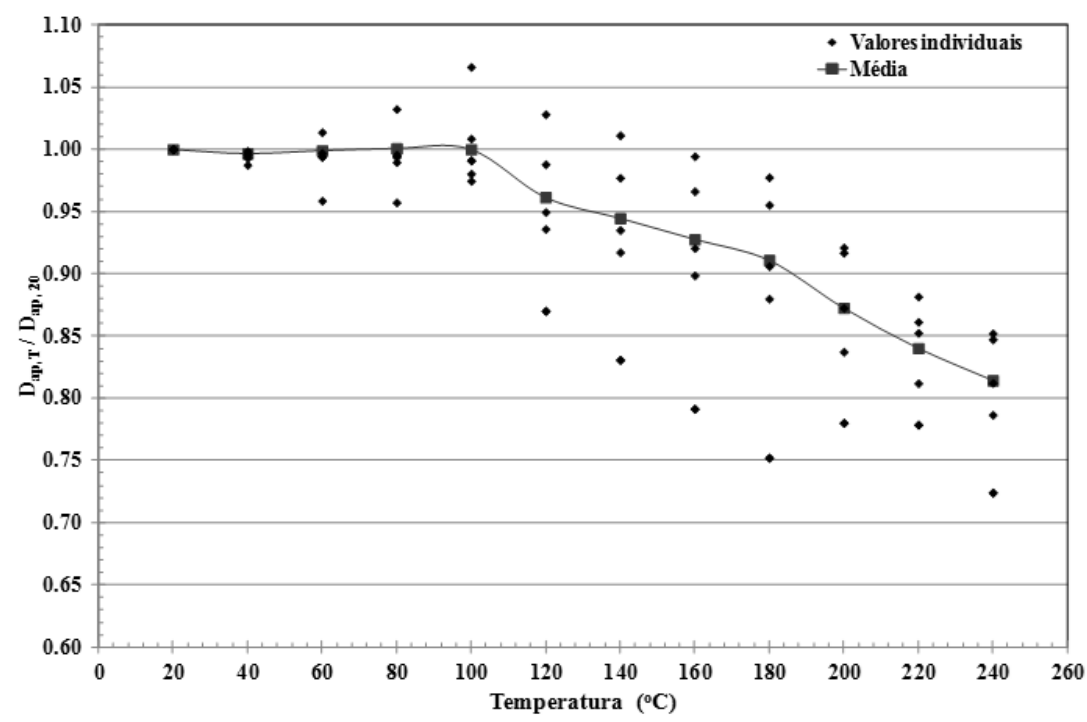

FIGURA 4: Massa específica relativa em função do aumento da temperatura.

FIGURE 4: Relative Specific Gravity according to the increasing temperature.

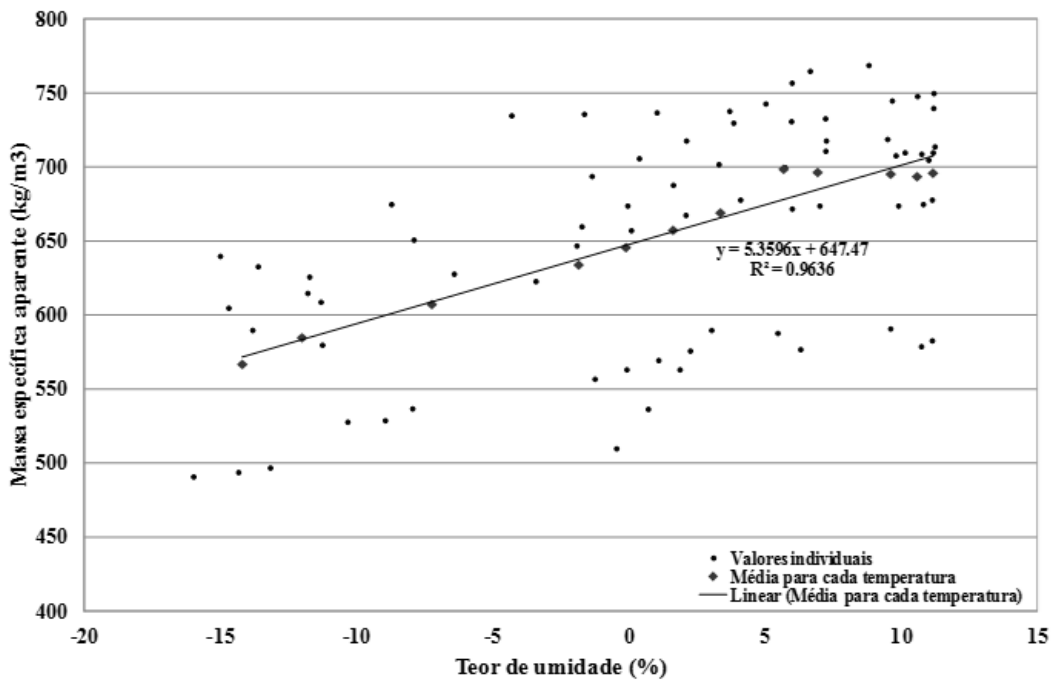

FIGURA 5: Massa específica relativa em função do teor de umidade.

FIGURE 5: Specific Gravity versus moisture content.

variação da massa específica em função da variação da umidade. Nota-se que existe uma correlação linear, altamente significante, entre os valores médios. Não foi possível explicar este fato com os dados disponíveis.

\section{Resistência à compressão paralela às fibras}

A resistência à compressão paralela às fibras em função da temperatura está apresentada na Tabela 3 e, como exemplo, é mostrado na Figura 6 o aspecto visual de cada um dos CPs da viga 2 após serem submetidos às temperaturas de $20^{\circ} \mathrm{C}$ a $240^{\circ} \mathrm{C}$, na qual se observam a variação da coloração da madeira e alguns modos de ruptura pós-ensaio de compressão.

A partir de uma análise visual dos modos de ruptura dos CPs em função da temperatura e dos apresentados na ASTM D 143 (2009) (Figura 7) até a temperatura de $100^{\circ} \mathrm{C}$ se detectou na maioria dos CPs o tipo "d". Para as temperaturas entre 120 e $180^{\circ} \mathrm{C}$, os tipos "e" e "b". Para temperaturas acima de $180^{\circ} \mathrm{C}$ não se detectou nenhum dos tipos apresentados pela ASTM D 143 (2009). A ruptura 
TABELA 3:Resistência à compressão paralela às fibras em função da temperatura.

TABLE 3: Compressive strength parallel to the fibers according to the temperature.

\begin{tabular}{ccccccccc}
\hline Temperatura $\left({ }^{\circ} \mathrm{C}\right)$ & $\begin{array}{c}\mathrm{f}_{\mathrm{c} 0}(\mathrm{MPa}) \\
\text { Viga } 1\end{array}$ & $\begin{array}{c}\mathrm{f}_{\mathrm{c} 0}(\mathrm{MPa}) \\
\text { Viga } 2\end{array}$ & $\begin{array}{c}\mathrm{f}_{\mathrm{c0}}(\mathrm{MPa}) \\
\text { Viga 3 }\end{array}$ & $\begin{array}{c}\mathrm{f}_{\mathrm{c0}}(\mathrm{MPa}) \\
\text { Viga 4 }\end{array}$ & $\begin{array}{c}\mathrm{f}_{\mathrm{c} 0}(\mathrm{MPa}) \\
\text { Viga } 5\end{array}$ & $\begin{array}{c}\mathrm{f}_{\mathrm{c} 0}(\mathrm{MPa}) \\
\text { Viga } 6\end{array}$ & $\begin{array}{c}\mathrm{f}_{\mathrm{c} 0}(\mathrm{MPa}) \\
\text { Média }\end{array}$ & $\begin{array}{c}\mathrm{f}_{\mathrm{c} 0}(\mathrm{MPa}) \\
\text { Desv.Pad. }\end{array}$ \\
\hline 20 & 64 & 49 & 71 & 44 & 62 & 66 & 59,33 & 10,50 \\
40 & 67 & 49 & 70 & 54 & 67 & 57 & 60,67 & 8,50 \\
60 & 65 & 57 & 72 & 55 & 70 & 52 & 61,83 & 8,33 \\
80 & 70 & 58 & 74 & 57 & 68 & 72 & 66,50 & 7,26 \\
100 & 73 & 64 & 81 & 63 & 70 & 66 & 69,50 & 6,77 \\
120 & 72 & 57 & 79 & 62 & 72 & 72 & 69,00 & 8,00 \\
140 & 79 & 49 & 80 & 58 & 74 & 71 & 68,50 & 12,41 \\
160 & 80 & 58 & 80 & 49 & 76 & 60 & 67,17 & 13,21 \\
180 & 61 & 65 & 83 & 59 & 73 & 51 & 65,33 & 11,27 \\
200 & 59 & 46 & 70 & 56 & 65 & 50 & 57,67 & 9,00 \\
220 & 49 & 39 & 52 & 32 & 47 & 47 & 44,33 & 7,42 \\
240 & 26 & 44 & 54 & 33 & 43 & 42 & 40,33 & 9,69 \\
\hline
\end{tabular}
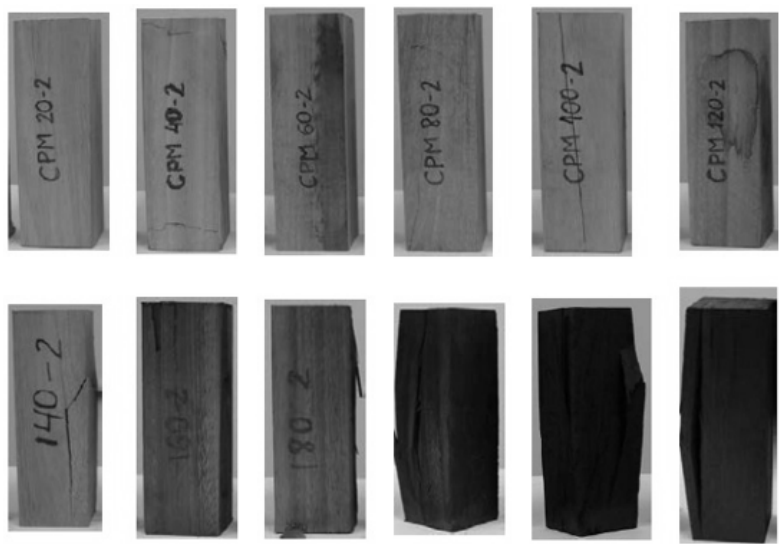

FIGURA 6: Variação na coloração da madeira e modos de ruptura após o ensaio de compressão.

FIGURE 6: Variation in the wood color and rupture modes after the compression test.

em todos os casos ocorreu por explosão indicando uma fragilidade na união dos elementos anatômicos da madeira.

$\mathrm{Na}$ Figura 8 é apresentado o gráfico da resistência relativa em função da variação de temperatura.

Ao se analisar a Figura 8, comparando-a com a resistência relativa da madeira à temperatura ambiente com a de $100^{\circ} \mathrm{C}$ observa-se que ocorre um acréscimo de $19 \%$, em média e, após os $100^{\circ} \mathrm{C}$, o comportamento se inverte com decréscimo linear
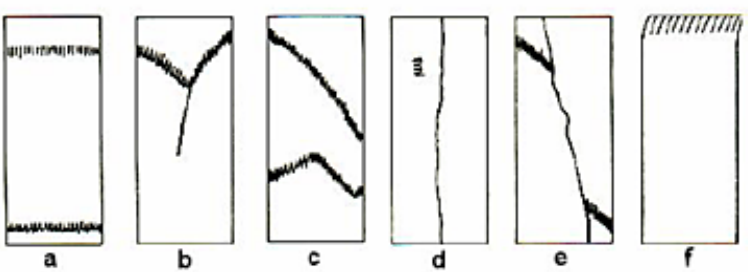

FIGURA 7:Modos derupturana compressão paralela às fibras: a) esmagamento horizontal; b) fenda radial ou tangencial; c) cisalhamento transversal; d) rachadura longitudinal; e) esmagamento e cisalhamento paralelo às fibras; f) esmagamento na extremidade. Fonte: ASTM D 143 (2009).

FIGURE 7: Modes of rupture in compression parallel to the grain: a) horizontal crushing, b) radial or tangential crack, c) transverse shear, d) Longitudinal crack e) crushing and shear parallel to the grains f) end crushing. Source: ASTM D 143 (2009).

até a temperatura de $180^{\circ} \mathrm{C}$, porém, ainda $10 \%$ maior do que a resistência à temperatura de $20^{\circ} \mathrm{C}$. A partir de $180^{\circ} \mathrm{C}$, a queda de resistência é elevada, chegando, na temperatura de $240^{\circ} \mathrm{C}$, a $32 \%$ menor do que a resistência na temperatura de $20^{\circ} \mathrm{C}$. Segundo Piazza et al. (2005), o aumento aparente de resistência é devido à diminuição da umidade. 


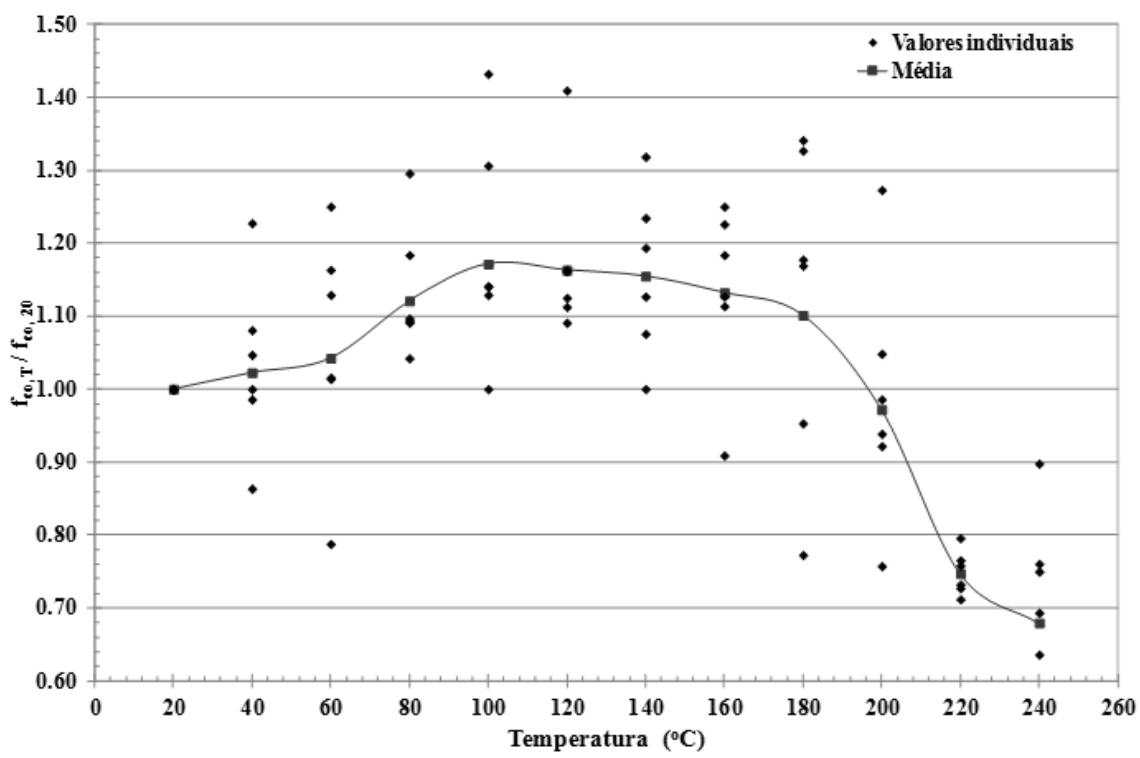

FIGURA 8: Resistência relativa à compressão paralela às fibras em função da temperatura.

FIGURE 8: Relative resistance to compression parallel to the fibers according to the temperature.

Já Kretschmann (2010) indica que para um teor de umidade constante e temperaturas abaixo de $150^{\circ} \mathrm{C}$, as propriedades de resistência mecânica diminuem linearmente com a temperatura. Visando explicar essa redução de resistência, White e Dietenberger (2001) e Rocha, Pérez e Cortez (2004) indicam que a biomassa lignocelulósica é uma mistura complexa de polímeros naturais de carboidratos conhecidos como celulose e hemicelulose, além de lignina, estando também presentes outras substâncias em pequenas quantidades como extrativos e cinzas, tornando a pirólise da madeira, um processo bastante complexo no qual a conversão térmica que ocorre implica na ruptura de ligações carbono-carbono e na formação de ligações carbono-oxigênio. Manríquez (2008) também encontrou que a resistência mecânica do paricá (Schizolobium amazonicum), diminuiu até $35,07 \%$ na temperatura de $230^{\circ} \mathrm{C}$ e Manríquez e Moraes (2009) indicam reduções na resistência à compressão paralela às fibras para diversas espécies

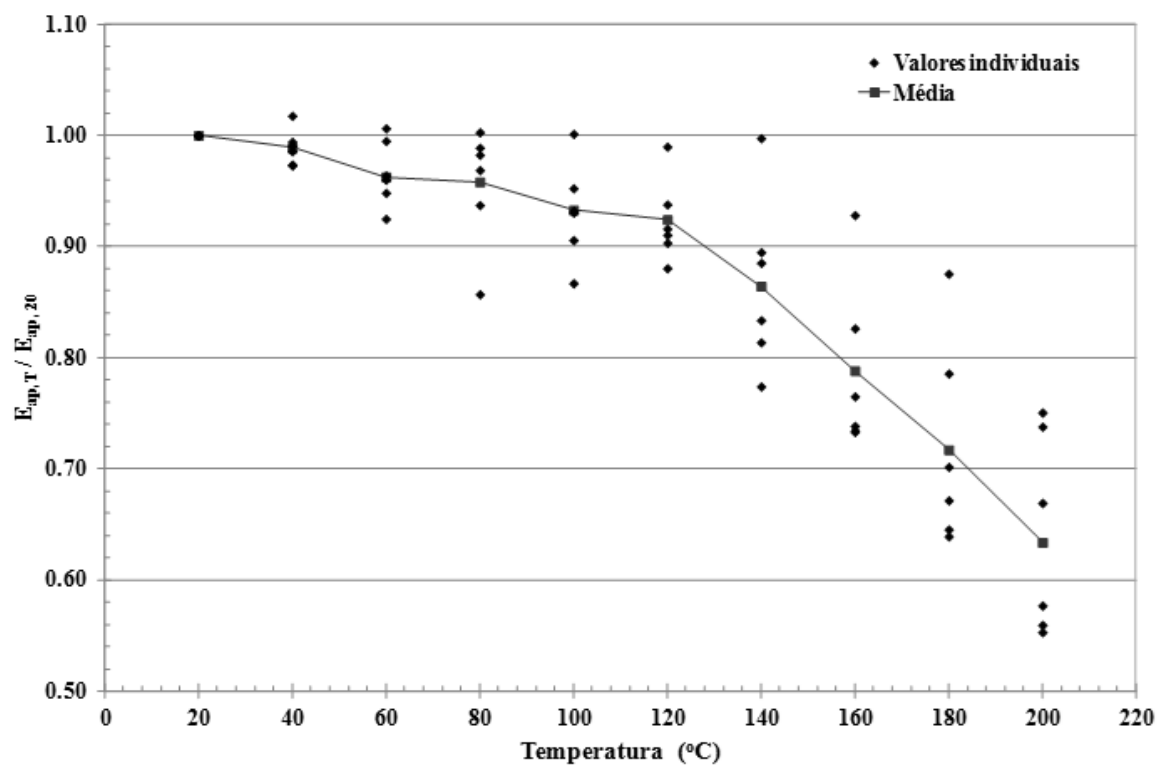

FIGURA 9: Módulo de elasticidade relativo à compressão paralela às fibras em função da temperatura. FIGURE 9: Elasticity modulus relative to compression parallel to the fibers as a function of temperature. 
de até $75 \%$.

\section{Módulo de elasticidade}

Na Tabela 4 apresentam-se os valores do módulo de elasticidade em função da temperatura e na Figura 9 tem-se o gráfico do módulo de elasticidade relativo em função da temperatura.

Analisando o gráfico da Figura 9 observa-se que o comportamento do módulo de elasticidade em função do aumento da temperatura é muito diferente do comportamento da resistência à compressão (Figura 8). O módulo de elasticidade diminui apenas aumenta a temperatura. Até $120^{\circ} \mathrm{C}$ existe uma diminuição linear não muito acentuada, em torno de $8 \%$. Já de $120^{\circ} \mathrm{C}$ até $200^{\circ} \mathrm{C}$ a diminuição também é linear, porém, bastante acentuada chegando a $38 \%$. Esse fato já foi constatado por Piazza, Tomasi e Modena (2005) e Manríquez e Moraes (2009).

$\mathrm{Na}$ Figura 10 é apresentado um gráfico relacionando à resistência relativa e o módulo de elasticidade relativa com a massa específica relativa. Observa-se que enquanto a massa específica relativa permanece 1 (até a temperatura de $100^{\circ} \mathrm{C}$ ) não existe uma correlação com a resistência e o módulo de elasticidade relativo, já para temperaturas acima de $100^{\circ} \mathrm{C}$ a correlação é altíssima. Este comportamento pode ser explicado devido ao fato de a massa específica relativa não sofrer praticamente nenhuma variação até a temperatura de $100^{\circ} \mathrm{C}$, como mostra a Figura 4, e a resistência relativa aumentar consideravelmente devido à diminuição do teor de umidade com o aumento da temperatura. Assim não existe esta correlação entre estas duas variáveis no intervalo de $20^{\circ} \mathrm{C}$ a $100^{\circ} \mathrm{C}$.

\section{CONCLUSÃO}

A madeira de híbridos de Eucalyptus grandis com Eucalyptus urophylla apresenta uma diminuição linear do teor de umidade com o aumento da temperatura até $160^{\circ} \mathrm{C}$. Destacamse duas retas, a primeira de $20^{\circ} \mathrm{C}$ até $65^{\circ} \mathrm{C}$ com pequeno decréscimo do teor de umidade, chegando a $4 \%$ e a segunda de $65^{\circ} \mathrm{C}$ até $160^{\circ} \mathrm{C}$ com perda mais acentuada chegando a $0 \%$ de teor de umidade. Acima de $160^{\circ} \mathrm{C}$, não apresentando mais umidade, a madeira perde apenas material orgânico, chegando a $20 \%$ de perda de massa quando atinge uma temperatura de $240^{\circ} \mathrm{C}$. A massa específica praticamente permanece constante até a temperatura de $100^{\circ} \mathrm{C}$ indicando que a perda de volume devido à diminuição da umidade é compensada pelo aumento de volume devido à dilatação térmica. A partir da temperatura de $100^{\circ} \mathrm{C}$ até $240^{\circ} \mathrm{C}$, a massa específica decresce linearmente chegando a $20 \%$. A resistência à compressão paralela às fibras aumenta linearmente com a temperatura, chegando a $19 \%$ na temperatura de $100^{\circ} \mathrm{C}$ e após esta temperatura o comportamento se inverte com decréscimo linear até a temperatura de $180^{\circ} \mathrm{C}$, porém, ainda $10 \%$ maior do que a resistência à temperatura de $20^{\circ} \mathrm{C}$. A partir de $180^{\circ} \mathrm{C}$, a queda de resistência é elevada, chegando, na temperatura de $240^{\circ} \mathrm{C}$ a $32 \%$ menor do que a resistência na temperatura de $20^{\circ} \mathrm{C}$. O

TABELA 4: Módulo de elasticidade paralelo às fibras em função da temperatura.

TABLE 4: Elastic modulus parallel to the grains as a function of temperature.

\begin{tabular}{ccccccccc}
\hline \multirow{2}{*}{ Temperatura $\left({ }^{\circ} \mathrm{C}\right)$} & $\begin{array}{c}\mathrm{E}_{\mathrm{c0}}(\mathrm{MPa}) \\
\text { Viga 1 }\end{array}$ & $\begin{array}{c}\mathrm{E}_{\mathrm{c0}}(\mathrm{MPa}) \\
\text { Viga 2 }\end{array}$ & $\begin{array}{c}\mathrm{E}_{\mathrm{c0}}(\mathrm{MPa}) \\
\text { Viga 3 }\end{array}$ & $\begin{array}{c}\mathrm{E}_{\mathrm{c0}}(\mathrm{MPa}) \\
\text { Viga 4 }\end{array}$ & $\begin{array}{c}\mathrm{E}_{\mathrm{c0}}(\mathrm{MPa}) \\
\text { Viga 5 }\end{array}$ & $\begin{array}{c}\mathrm{E}_{\mathrm{c0}}(\mathrm{MPa}) \\
\text { Viga 6 }\end{array}$ & $\begin{array}{c}\mathrm{E}_{\mathrm{c0}}(\mathrm{MPa}) \\
\text { Média }\end{array}$ & $\begin{array}{c}\mathrm{E}_{\mathrm{c0}}(\mathrm{MPa}) \\
\text { Desv.Pad. }\end{array}$ \\
\hline 20 & 12715 & 8758 & 12925 & 8922 & 11671 & 12248 & 11207 & 1884 \\
40 & 12371 & 8632 & 12585 & 8869 & 11558 & 12463 & 11080 & 1841 \\
60 & 11759 & 8813 & 12408 & 8877 & 11067 & 11804 & 10788 & 1564 \\
80 & 12573 & 8208 & 12960 & 8643 & 10002 & 12033 & 10737 & 2065 \\
100 & 12731 & 8150 & 12309 & 8317 & 10118 & 11093 & 10453 & 1950 \\
120 & 11924 & 7911 & 11379 & 8172 & 10625 & 12123 & 10356 & 1868 \\
140 & 10600 & 7753 & 10518 & 7984 & 9034 & 12216 & 9684 & 1731 \\
160 & 9728 & 6432 & 9545 & 7372 & 8557 & 11368 & 8834 & 1773 \\
180 & 8128 & 6146 & 8681 & 7010 & 7533 & 10723 & 8037 & 1583 \\
200 & 7113 & 5861 & 7457 & 6697 & 6455 & 9038 & 7104 & 1095 \\
\hline
\end{tabular}




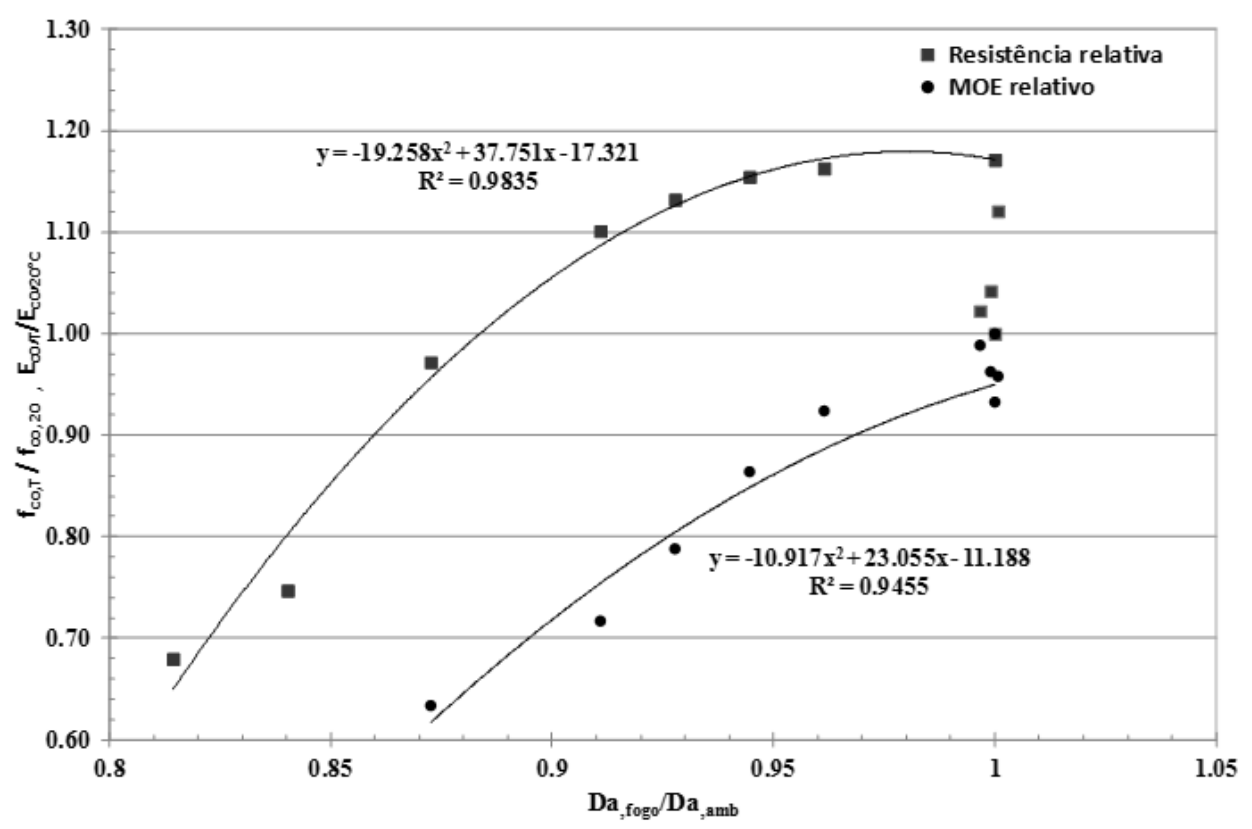

FIGURA 10: Resistência e módulo de elasticidade relativo à compressão paralela às fibras em função da massa específica relativa.

FIGURE 10: Resistance and elasticity modulus relative to the parallel compression to the grains according to the relative specific gravity.

comportamento do módulo de elasticidade em função do aumento da temperatura é muito diferente do comportamento da resistência à compressão. $\mathrm{O}$ módulo de elasticidade diminui apenas aumenta a temperatura. Até $120^{\circ} \mathrm{C}$ existe uma diminuição linear não muito acentuada, em torno de $8 \%$. Já de $120^{\circ} \mathrm{C}$ até $200^{\circ} \mathrm{C}$, a diminuição também é linear, porém, bastante acentuada chegando a 38\%. A resistência e o módulo de elasticidade relativo até a temperatura de $100^{\circ} \mathrm{C}$ não têm uma correlação significativa com a massa específica relativa, já para temperaturas acima de $100^{\circ} \mathrm{C}$, a correlação é altíssima.

\section{AGRADECIMENTOS}

À FAPEMIG - Fundação de Amparo à Pesquisa do Estado de Minas Gerais e ao $\mathrm{CNPq}-$ Conselho Nacional de Desenvolvimento Científico e Tecnológico, pelo auxílio financeiro.

\section{REFERÊNCIAS BIBLIOGRÁFICAS}

AMERICAN SOCIETY FOR TESTING AND MATERIALS. Standard methods of testing small clear specimens of timber. ASTM D-143-94. Annual book of ASTM Standards, 2007. ASSOCIAÇÃO BRASILEIRA DE NORMAS
TÉCNICAS. NBR 7190: Projeto de estruturas de madeira. Rio de Janeiro 1979.

NBR 14432: Exigências de resistência ao fogo de elementos construtivos de edificações Procedimento. Rio de Janeiro 2001.

BAKAR, B. F. A.; HIZIROGLU, S.; TAHIR, P. Properties of some thermally modified Wood species. Materials and Design, v. 43, p. 348-355, 2012.

BUCHANAN, A. International developments in design for structural fire safety. Revista SulAmericana de Engenharia Estrutural, Passo Fundo, v. 1, n. 1, p. 51-78, 2004.

COSTA, C. N.; SILVA, V. P. Revisão histórica das curvas padronizadas de incêndio. In: SEMINÁRIO INTERNACIONAL NUTAU, 2006, São Paulo. Faculdade de Arquitetura e Urbanismo da Universidade de São Paulo. Anais... São Paulo, 2006.

GERHARDS, C. C. Effect of the moisture content and temperature on the mechanical properties of wood: an analysis of immediate effects. Wood and Fiber, Pennsylvania, USA, v. 14, n. 1, p. 4-36, 1982.

KNUDSON, R. M.; SCHNIEWIND, A. P. Performance of structural wood members exposed to fire. Forest Products Journal, USA, v. 25, n. 2 , 
p. 23-32, 1975.

KRETSCHMANN, D. E. Wood Handbook: wood as an engineering material. [s. 1.]: U.S. Department of Agriculture, Forest Service, Forest Products Laboratory, 2010.

MANRÍQUEZ F, M. J. Coeficientes de modificação das propriedades mecânicas da madeira devidos à temperatura. 2012. $259 \mathrm{f}$. Tese (Doutorado em Engenharia Civil) - Universidade Federal de Santa Catarina, Brasil, 2012.

Influência da temperatura sobre a resistência mecânica do paricá. 2008. 164 f. Dissertação (Mestrado em Construção Civil) - Universidade Federal de Santa Catarina, Brasil, 2008.

MORAES, P. D. Comportamento da madeira a temperaturas elevadas. Ambiente construído, v. 9, n. 4, p. 157-174, 2009.

PIAZZA, M.; TOMASI, R.; MODENA, R. Strutture in legno. Materiale, calcolo e progetto secondo Le nuove normative europee. Milano: Biblioteca Tecnica Hoepli, 2005. 736 p.

PINHEIRO, P. C. C.; SÈYE, O. Influência da temperatura da carbonização nas propriedades do carvão vegetal de Eucalyptus. In: CONGRESSO ANUAL DA ASSOCIAÇÃO BRASILEIRA DE METALURGIA E MATERIAIS, 53., 1998, Belo Horizonte. Anais... Belo Horizonte, 1998.

PINTO, E. M. Determinação de um modelo de taxa de carbonização transversal à grã para o Eucalyptus citriodora e Eucalyptus grandis. 2005. 138 f. Tese (Doutorado em Ciência e Engenharia de
Materiais) - Universidade de São Paulo, São Carlos, 2005.

PURKISS, J. A. Fire safety engineering design of structures. Boston: Butterworth-Heinemann, 2007. $387 \mathrm{p}$.

RAAD, T. J.; PINHEIRO, P. C. C.; YOSHIDA, M. I. Equação geral de mecanismos cinéticos da carbonização do Eucaliptus spp. Cerne, Lavras, v. 12, n. 2, p. 93-106, 2006.

ROCHA, J. D.; PÉREZ, J. M.; CORTEZ, L. A. B. Aspectos teóricos e práticos do processo de pirólise de biomassa. Curso "Energia na Indústria de Açúcar e Álcool”. Itajubá: UNIFEI, 2004. 22 p.

SIAU, J. F. Transport processes in wood. 1. ed. Berlin: Springer-Verlag, 1984. 245 p.

SKAAR, C. Wood-Water Relations. 1. ed. Berlin: Springer, 1988. $283 \mathrm{p}$.

THOMAS, G. C. Fire resistance of light timber framed walls and floors. 1996. $176 \mathrm{f}$. Tese (Phd in Fire Engineering) - University of Canterbury, New Zealand's South Island, 1996.

SCHAFFER, E. L. Charring rate of selectad woods: transverse to grain. [s. 1.]: U.S. Department of Agriculture. Forest Service. Forest Products Laboratory, 1967.

WHITE, R. H.; DIETENBERGER, M. A. Wood products: thermal degradation and fire. [s. 1.]: Elsevier Science, 2001. p. 9712-9716.

YOUNG, S. A.; CLANCY, P. Compression mechanical properties of wood at temperatures simulating fire conditions. Fire and Materials, v. 25, p. 83-93, 2001. 